\title{
Quantitation of Virus Particles \\ by Negative Staining for TEM
}

\section{$10 \mid$}

While many/most of the articles in this series have related to electron microscopy, we are interested in publishing them relating to all microscopy techniques. Contributions would be greatly appreciated and may be sent to Phil Oshel by:

eMail: oshel@ux1.cso.uiuc.edu

Snail Mail: Station A

PO Box 5037

Champaign IL 61825-5037

\section{Hematoxylin Stain for Epon Sections:}

Other than toluidine blue, we also use a variation on the hematoxylin theme for our epon sections.

Solutions for Regaud's hematoxylin (Heidenhain's hematoxylin can be bought premade, and works equally well)

Iron Alum Solution:

$5 \%$ ferric ammonium sulfate ( $5 \mathrm{~g}$ per $100 \mathrm{~mL}$ distilled water)

Discard any crystals which have turned yellow.

Hematoxylin Solution:

$10 \mathrm{~g}$ hematoxylin

$100 \mathrm{~mL}$ ethanol

$100 \mathrm{~mL}$ glycerin

distilled water

Dissolve the hematoxylin in ethanol. Add the glycerin and make up to $1000 \mathrm{~mL}$ with distilled water. Cover with aluminum foil and let ripen for AT LEAST one month. Longer is better, usually 4 months to a year. 0.2 to $0.25 \mathrm{~g} \mathrm{NaIO}_{3}$ can be added to ripen the solution in 24 to 48 hours. MT)

Slides are preheated and stained on a hot plate that is maintained between $80^{\circ}$ and $90^{\circ} \mathrm{C}$

Coat the sections with the iron alum solution for 2-10 min depending on the tissue type, thickness, etc

Rinse VERY WELL with distilled water. Repeat the process on the hot plate with the hematoxylin staining solution using the same time interval as the iron alum. Because the "staining" only appears at this step, you may need to test a few slides to determine ideal timing.

After rinsing well again with distilled water, flood the slides on the hot plate with TAP water to differentiate and let sit for $3 \mathrm{~min}$.

Rinse briefly in distilled water and dry.

We stain mainly tissue samples and while the nuclear detail is great, the cytoplasm is not completely clear as membranes and other details are quite apparent. One of our staff members, Dr. C.P. Leblond, tells me he has tried a number of counterstains but finds he get better results without them. (this is likely the result of using Fe as the mordant, since elements other than nuclei, such as myelin and membranes, are stained. Using Al for the mordant would give clearer cytoplasm.-MT)

Prestaining like this allows the slides to then go through autoradiography without interfering with the emulsion and has worked for us irregardless of the fixative used

Pat Hales, McGill University
1. A selected volume $(100 \mu \mathrm{L})$ of supernatant containing virus particles is mixed with a selected volume $(100 \mu \mathrm{L})$ of polystyrene latex beads of known concentration (about $10^{\mathrm{a}}$ beads per $\mathrm{mL}$ ) and a size between $100-200 \mathrm{~nm}$ in diameter.

2. The mixture is placed in a Beckman Airfuge $240 \mu \mathrm{L}$-tube. A Formvar and carbon-coated grid is inserted into the bottom of the microtubes. ${ }^{*}$ The tubes are placed in a Airfuge A-100 fixed angle rotor (30x) and centrifuged at $20 \mathrm{psi}$ $(120,000 \mathrm{~g})$ for 5 minutes

3. The grids are recovered with fine self-closing tweezers. dried with bibulous paper, stained 1 minute with phosphotungstic acid (PTA $3 \% \mathrm{pH} \mathrm{6.0)} \mathrm{and} \mathrm{dried}$ again with bibulous paper. Samples are visualized under a transmission electron microscope with an appropriate magnification.

4 Or two different grids, 250-500 particles (latex beads or virus particles) are counted from at least five different areas on each grid. Since the ratio of the two types of particles in the suspension can be determined from the ratio of the volumes added, and the concentration of latex particles is known, the concentration of viral particles can be calculated.

Viral particle concentration $=$ (particles/mL)

virus count

The level of sensitivity of this procedure is between $10^{6}$ and $10^{10}$ particles per $\mathrm{mL}$. Less than $10^{6}$, there is not enough virus to get a realistic count and more than $10^{10}$, there are too many particles to well differentiate them. This procedure is used principally to quantify Retrovirus type-A and $-C$ particles in cells supernatant, but can be used for any virus particles

${ }^{\star}$ R. Alain et al. J.Virol. Meths. 16(1987), 209-216

Robert Alain, Institut Armand-Frapplier

\section{Suggestions to Improve the Lifetime of EDX Detector Windows}

As the manufacturer of ultrathin windows for EDX detectors, Moxtek can offer the following suggestions to improve window lifetimes

1) Slow down the chamber venting.

2) Retract the detector before venting (if the system has the capability)

3) Cover the detector with a plastic bag when it is not going to be used. The most common field failure is what we call "bullet holes". These occur when particulates in the chamber are swirled up during venting and impact the surface of the window. The first three suggestions help prevent this.

4) Never pump down your specimen chamber with a x-ray detector attached. Make sure you cool down the detector before pumpdown. Most of the detector systems have a molecular sieve or other desiccant material in the dewar system to trap any residual gases. When the detector is warmed up, this material can outgas these trapped gases, and if the system is then pumped down, the window is reverse-pressured. These windows are not designed to tolerate any back pressure.

5) Never overheat the window by exposure to a hot stage. The instrument manufacturer should be able to recommend how to operate the system to minimize heat transfer to the detector window.

6) Consult the EDX manufacturer for specific instructions for your system. Every detector/microscope combination is unique, and they should have some experience that will help you.

There are various thickness of windows available, but the above suggestions apply regardless of the thickness. If there are continual failures with the thinnest windows, you should be able to get a thicker window from the EDX manufacturer.

Clark Turner, Moxtek Inc. 